\title{
34 Nahrungsmittelunverträglichkeiten
}

\author{
(c) Springer-Verlag GmbH Deutschland, ein Teil von Springer Nature 2018 \\ D. Mathias, Fit und gesund von 1 bis Hundert \\ https://doi.org/10.1007/978-3-662-56307-6_34
}

Verdauungsprozesse sind zeitintensive Vorgänge (Magen 2-6 Std., Dünndarm 6-8 Std., Dickdarm 10-20 Std. und Mastdarm 20-60 Std.). Diese Zeiten variieren individuell und hängen sehr stark von der Nahrungszusammensetzung ab. Trotz der langen Dauer kann es passieren, dass nicht alle mit der Nahrung aufgenommenen Stoffe in unserem Körper optimal verarbeitet werden. Wenn es in diesem Zusammenhang immer wieder zu Problemen kommt, spricht man von Nahrungsmittelunverträglichkeiten. Es wird geschätzt, dass ca. 1-2 \% aller Menschen an einer solchen Verwertungsintoleranz von Nahrungsmitteln leiden. Aber etwa $20 \%$ der Menschen geben an, dass sie glauben, bestimmte Nahrungsmittel nicht zu vertragen.

Viele unverträgliche Nahrungsmittel bewirken immunologische Reaktionen und damit die sogenannten Nahrungsmittelallergien (Goodman 2013; Sicherer und Sampson 2014). Oft sind diese auch pollenassoziiert. Das Immunglobulin E ist dabei die zentrale Schaltstelle. Häufiger Kontakt mit den entsprechenden Allergenen führt zur verstärkten Sensibilisierung. Auch die Zöliakie, bei der eine Gluten-Intoleranz vorliegt, ist von immunologischen Abläufen geprägt, hier spielen das IgA und gelegentlich das IgG die entscheidende Rolle. Etwa $1 \%$ der erwachsenen Bevölkerung in den Industriestaaten ist von dieser speziellen Erkrankung betroffen. Kindern sollten allein aus Gründen der Vorsicht potenziell allergene Lebensmittel nicht vorenthalten werden. Denn deren regelmäßiger Verzehr führt bei ihnen meist zu einer dauerhaften immunologischen Toleranz (Du Toit et al. 2015).

Die sog. resorptionsbedingte Intoleranz ist eine nicht-immunologische Reaktion. Hier können die betroffenen Personen bestimmte Nahrungs- bestandteile schlecht oder gar nicht resorbieren. Solche Defekte sind oft angeboren, manchmal aber auch erworben. Hierzu gehört die Fructose-Intoleranz. Ferner sind Enzymmängel oder Enzymdefekte verantwortlich dafür, dass manche Menschen einige Nahrungsbestandteile nicht komplett verdauen können. Beispiele sind die Laktose-Intoleranz ( $\triangleright$ Kap. 53) und die Histamin-Intoleranz.

Eine Reihe von Substanzen in Nahrungsmitteln entfalten pharmakologische Aktivitäten, wenn sie in größeren Mengen verzehrt werden. Dazu gehört z. B. das Coffein, aber auch biogene Amine wie das Tryptamin in Tomaten, Phenylethylenamin in Schokolade, Serotonin in Bananen und Nüssen sowie Tyramin in reifem Käse und ebenfalls in Schokolade.

Schließlich gibt es pseudoallergische Reaktionen auf Nahrungsmittelzusatzstoffe. Das klinische Bild, begleitet von einer Degranulierung der Mastzellen im Immunsystem, ähnelt dem der Allergien. Häufige Auslöser sind u. a. die Benzoesäure in Konservierungsstoffen, Salicylate in Aspirin, Äpfeln und Aprikosen, Lecithin in Emulgatoren, Lektine in Bohnen oder Essigsäure und Zitronensäure in Säuerungsmitteln.

Die Symptome der Nahrungsmittelunverträglichkeiten zeigen sich meist an der Haut und den Schleimhäuten. Es kann jedoch auch die Lunge, das Herz-Kreislauf-System oder der Magen-DarmTrakt betroffen seien. Juckreiz, Heiserkeit, Kratzen im Hals, Asthma, Schwellungen der Mundschleimhaut, Übelkeit, Koliken, Durchfälle, Hypotonien, Extrasystolen oder Tachykardien sind häufige Symptome. Wenn solche Beschwerden wiederholt nach einer Mahlzeit auftreten, lassen sich relativ leicht die verursachenden Lebensmittel herausfinden. Sie sind dann dauerhaft zu meiden. 